\title{
Prognostic Scoring Index for Patients with Metastatic Pancreatic Adenocarcinoma
}

\author{
Hyung Soon Park, MD \\ Hye Sun Lee, PhD² \\ Ji Soo Park, MD \\ Joon Seong Park, MD, PhD \\ Dong Ki Lee, MD, $\mathrm{PhD}^{4}$ \\ Se-Joon Lee, MD, $\mathrm{PhD}^{4}$ \\ Dong Sup Yoon, MD, PhD ${ }^{4}$ \\ Min Goo Lee, MD, PhD 1 \\ Hei-Cheul Jeung, MD, $P h D^{4,5}$
}

\author{
${ }^{1}$ Department of Pharmacology and \\ Brain Korea 21 Plus Project for \\ Medical Sciences, \\ ${ }^{2}$ Biostatistics Collaboration Unit, \\ ${ }^{3}$ Cancer Prevention Center, \\ Yonsei Cancer Center, \\ ${ }^{4}$ Pancreatobiliary Cancer Clinic, \\ Gangnam Severance Hospital, \\ ${ }^{5}$ Songdang Institute for Cancer Research, \\ Yonsei University College of Medicine, \\ Seoul, Korea
}

\author{
Correspondence: Hei-Cheul Jeung, MD, PhD, \\ Division of Medical Oncology, \\ Department of Internal Medicine, \\ Gangnam Severance Hospital, \\ Yonsei University College of Medicine, \\ 211 Eonju-ro, Gangnam-gu, Seoul 06273, Korea \\ Tel: 82-2-2019-1242 \\ Fax: 82-2-3463-3882 \\ E-mail: jeunghc1123@yuhs.ac \\ Received October 20, 2015 \\ Accepted January 14, 2016 \\ Published Online February 3, 2016
}

\section{Purpose}

This study focused on implementation of a prognostic scoring index based on clinico-laboratory parameters measured routinely on admission in metastatic pancreatic cancer patients.

\section{Materials and Methods}

Records from 403 patients of metastatic disease were analyzed retrospectively. Continuous variables were dichotomized according to the normal range or the best cut-off values statistically determined by Contal and O'Quigley method, and then analyzed in association with prognosis-overall survival (OS), using Cox's proportional hazard model. Scores were calculated by summing the rounded chi-square scores for the factors that emerged in the multivariate analysis.

\section{Results}

Performance status, hemoglobin, leucocyte count, neutrophil-lymphocyte ratio, and carcinoembryonic antigen were independent factors for OS. When patients were divided into three risk groups according to these factors, median survival was 11.7, 6.2, and 1.3 months for the low, intermediate, and high-risk groups, respectively $(p<0.001)$. Palliative chemotherapy has a significant survival benefit for low and intermediate-risk patients (median OS; 12.5 months vs. 5.9 months, $p<0.001$ and 8.0 months vs. 2.0 months, $\mathrm{p}<0.001$, respectively).

\section{Conclusion}

We advocate the use of a multivariable approach with continuous variables for prognostic modeling. Our index is helpful in accurate patient risk stratification and may aid in treatment selection.

\section{Introduction}

Pancreatic ductal adenocarcinoma (PDAC) is the fourth most common cause of cancer-related mortality in the Western world. Only 5\%-20\% of patients are candidates for curative pancreatectomy and most cases of PDAC are diagnosed
Key words

Pancreatic neoplasms, Prognosis, Research design, Survival 
superior outcomes compared with gemcitabine alone [1] However, these new regimens are associated with considerable toxicities and the long-term survival remains unsatisfactory.

Identification of prognostic factors is crucial in guiding personalized medicine. Such factors help patients to avoid unnecessary toxicity and complications from aggressive treatment and can further facilitate stratification for clinical trials. Many studies have examined various preoperative/ postoperative factors predictive of treatment outcomes [2]. However, their significance is still questionable, as most have been identified based on small sample sizes with inadequate statistical power. Widely suggested parameters are derived from functional and nutritional status, distant metastasis, and laboratory findings including C-reactive protein (CRP), carcinoembryonic antigen (CEA), and carbohydrate antigen 19-9 (CA 19-9) [3]. However, the role of these parameters is not affirmed, and the risk of bias subsists when the choice of cutoff for the continuous variables is random, leading to the use of different cutoff points across multiple studies, which impedes direct comparison. In addition, regarding advanced disease which is unresectable or metastatic, few risk scoring indices were available, and the majority focused on prediction after chemotherapy - particularly gemcitabine [4]. However, it is more important in the clinical field to discriminate patients who might benefit from chemotherapy from those who cannot. Therefore, the aim of this study is to determine prognostic values of clinic-laboratory parameters routinely evaluated at the time of diagnosis, leading to implementation of a scoring index for use in effective identification of different risk patient groups.

\section{Materials and Methods}

\section{Patients}

From July 2006 to April 2014, 482 patients were diagnosed with pancreatic cancer at Gangnam Severance Hospital, Seoul, Korea. Diagnosis was made from surgical excision, tissue biopsy, or cytology. Inclusion criteria for further analysis were as follows: (1) age $>18$ years, (2) histologically confirmed diagnosis of PDAC, (3) metastatic disease at the time of diagnosis or systemic recurrence after curative resection, and (4) available electronic medical records (including treatment information).

Exclusion criteria were as follows: (1) brain or leptomeningeal metastasis; (2) locally advanced disease who received definitive chemoradiotherapy; and (3) other histology types such as cystic neoplasms, neuroendocrine tumors, or lymphomas; or (4) synchronous advanced malignancies. A total of 403 patients fulfilled the inclusion criteria and were analyzed retrospectively. The institutional review board approved this retrospective study and the requirement to obtain informed consent was waived.

\section{Data collection}

The following baseline data were recorded at the time of diagnosis: age, sex, previous pancreatectomy, tumor location, Eastern Cooperative Oncology Group (ECOG) performance status (PS), metastatic site(s), presence of diabetes mellitus, presence of thromboembolism, and body mass index (BMI). Hematological and blood chemistry values included hemoglobin, white blood cell (WBC) count, serum protein, albumin, total bilirubin, cholesterol, blood urea nitrogen (BUN), alkaline phosphatase (ALP), aspartate aminotransferase (AST), alanine aminotransferase (ALT), CRP, CEA, and CA 19-9. Neutrophil-to-lymphocyte ratio (NLR) was calculated by dividing absolute neutrophil count by absolute lymphocyte count.

Computed tomography (CT) of chest and abdominopelvis, radionuclide bone scan, and ${ }^{18} \mathrm{~F}$-fluorodeoxyglucose positron emission tomography were performed for evaluation of distant metastasis. Radiological detection of peritoneal carcinomatosis relied on the following findings evaluated by independent radiologist(s): thickening and nodular enhancement of peritoneal reflections, multiple soft tissue nodules, stranding and thickening of the omentum (omental cake), stranding and distortion of the small bowel mesentery, and ascites, especially if loculated. The presence of ascites was inferred from evidence of paracentesis and malignant cell detection in cytology examination, or from suspicious fluid collection on CT or ultrasonogram.

\section{Statistical analysis}

Hematological and blood chemistry values were initially recorded as continuous variables and later transformed into categorical variables according to the upper normal values (hemoglobin, WBC, serum protein, albumin, bilirubin, BUN, ALP, AST, and ALT) [5] or the best cutoff point (NLR, CRP, CEA, and CA 19-9) determined using the Contal and O'Quigley method, which calculates the maximizing hazard ratio (HR) based on log rank statistics and estimates the best cut-off value [6].

Overall survival (OS) was defined as the time from the date of diagnosis until death from any cause. Survival curves were generated using the Kaplan-Meier method and compared using the log-rank test. Univariate analysis was performed to determine the association of the following prognostic factors with OS: age, sex, previous pancreatec- 
tomy, tumor location, PS, diabetes mellitus, liver metastasis, peritoneal carcinomatosis, thromboembolism, BMI, hemoglobin, WBC count, NLR, serum protein, albumin, bilirubin, BUN, ALP, AST, ALT, CRP, CEA, and CA 19-9. A stepwise multivariate analysis with Cox's proportional hazard model was performed using significant factors in the univariate analysis. HRs, 95\% confidence intervals (CIs), and chi-sqaure scores were obtained for all regressions.

A risk scoring system was devised by summing the rounded chi-sqaure scores of the independent prognostic factors identified in the multivariate analysis. Finally, patients were grouped according to the best cutoff value as calculated by K-adaptive partitioning, which calculates the best cutoff value by selection of maximizing the sum of pairwise logrank statistics [7]. Discriminatory power of the scoring system was assessed using Harrell's c-index [8]. A p-value of $<0.05$ was considered statistically significant.

Statistical analyses were performed using PASW Statistics ver. 18.0 (SPSS Inc., Chicago, IL), SAS ver. 9.2 (SAS Institute Inc., Cary, NC), and R ver. 3.1.3 (kaps Add-on Package; Institute for Statistics and Mathematics, Vienna, Austria; http://www.R-project.org).

\section{Results}

The median follow-up period was 7.9 months (range, 0.1 to 70.5 months) from diagnosis. Baseline patient characteristics are shown in Table 1 . The median age was 66 years, and 198 patients $(49.1 \%)$ were men. Sixty-two patients $(15.4 \%)$ underwent curative resection, 217 patients $(53.8 \%)$ had an ECOG PS of $0-1$, and $178(44.2 \%)$ and $79(19.6 \%)$ patients had liver metastasis and peritoneal carcinomatosis at the time of diagnosis, respectively. The median value of NLR was 2.92, and the median value of CEA and CA $19-9$ was $4.4 \mathrm{ng} / \mathrm{mL}$ and $224.5 \mathrm{U} / \mathrm{mL}$, respectively.

\section{Survival analysis}

Median OS for total patients was 8.2 months (95\% CI, 7.3 to 9.1 ). The statistically determined best cutoff points were as follows: 3.0 for NLR, $6.0 \mathrm{mg} / \mathrm{L}$ for CRP, $7.0 \mathrm{ng} / \mathrm{mL}$ for CEA, and 453.0 U/mL for CA 19-9. Significantly extended survival was observed in patients with low NLR levels compared with high NLR (median OS, 11.3 months vs. 4.7 months; $\mathrm{p}<0.001$ ) (Fig. 1A). Patients with low levels of CRP, CEA, or CA 19-9, respectively, also showed superior survival (median OS; CRP: 11.3 months vs. 5.0 months, $\mathrm{p}<0.001$; CEA: 10.9 months vs. 5.7 months, $\mathrm{p}<0.001$; CA 19-9: 10.3 months vs. 6.5 months, $\mathrm{p}<0.001$ ) (Fig. 1B-D).
Table 1. Patient characteristics

\begin{tabular}{|c|c|}
\hline Characteristic & No. $(\%)$ \\
\hline Age, median (range, yr) & $66(29-96)$ \\
\hline \multicolumn{2}{|l|}{ Sex } \\
\hline Male & $198(49.1)$ \\
\hline Female & $205(50.9)$ \\
\hline \multicolumn{2}{|l|}{ Previous pancreatectomy } \\
\hline Yes & $62(15.4)$ \\
\hline No & $341(84.6)$ \\
\hline \multicolumn{2}{|l|}{ Tumor location } \\
\hline Head & $226(56.1)$ \\
\hline Body/Tail & $177(43.9)$ \\
\hline \multicolumn{2}{|l|}{ ECOG performance status } \\
\hline $0-1$ & $217(53.8)$ \\
\hline 2 & $127(31.5)$ \\
\hline $3-4$ & $59(14.6)$ \\
\hline \multicolumn{2}{|l|}{ Diabetes mellitus } \\
\hline No & $260(64.5)$ \\
\hline Yes & $143(35.5)$ \\
\hline \multicolumn{2}{|l|}{ Liver metastasis } \\
\hline No & $225(55.8)$ \\
\hline Yes & $178(44.2)$ \\
\hline \multicolumn{2}{|l|}{ Peritoneal carcinomatosis } \\
\hline No & $324(80.4)$ \\
\hline Yes & 79 (19.6) \\
\hline \multicolumn{2}{|l|}{ Thromboembolism } \\
\hline No & $370(91.8)$ \\
\hline Yes & $33(8.2)$ \\
\hline BMI, median (range, kg/m²) & $22.05(13.1-35.6)$ \\
\hline \multicolumn{2}{|l|}{ Hematologic values, median (range) } \\
\hline Hemoglobin (g/dL) & $12.3(4.6-18.3)$ \\
\hline WBC count $(/ \mu \mathrm{L})$ & $6,560(2,280-23,430)$ \\
\hline NLR & $2.92(0.52-34.52)$ \\
\hline \multicolumn{2}{|c|}{ Blood chemistry values, median (range) } \\
\hline Protein $(g / d L)$ & $6.75(4.4-8.8)$ \\
\hline Albumin (g/dL) & $4.2(2.1-5.7)$ \\
\hline Bilirubin (mg/dL) & $0.7(0.1-30)$ \\
\hline BUN (mg/dL) & $13.5(4.2-116.2)$ \\
\hline ALP (IU / L) & $101(30-1,478)$ \\
\hline AST (IU/L) & $27(10-666)$ \\
\hline ALT (IU/L) & $25(5-552)$ \\
\hline CRP (mg/L) & $6.3(0.1-314.4)$ \\
\hline CEA (ng/mL) & $4.4(0.3-8,168.6)$ \\
\hline CA $19-9(\mathrm{U} / \mathrm{mL})$ & $224.55(0.5-20,850)$ \\
\hline \multicolumn{2}{|l|}{ Palliative treatment } \\
\hline Gemcitabine monotherapy & $87(21.6)$ \\
\hline Gemcitabine doublet therapy & $163(40.4)$ \\
\hline Fluoropyrimidine-based therapy & $36(8.9)$ \\
\hline RTx with chemotherapy & $29(7.2)$ \\
\hline No treatment & $88(21.8)$ \\
\hline
\end{tabular}

ECOG, Eastern Cooperative Oncology Group; BMI, body mass index; WBC, white blood cell; NLR, neutrophil-lymphocyte ratio; BUN, blood urea nitrogen; ALP, alkaline phosphatase; AST, aspartate aminotransferase; ALT, alanine aminotransferase; CRP, C-reactive protein; CEA, carcinoembryonic antigen; CA 19-9, carbohydrate antigen 19-9; RTx, radiotherapy. 


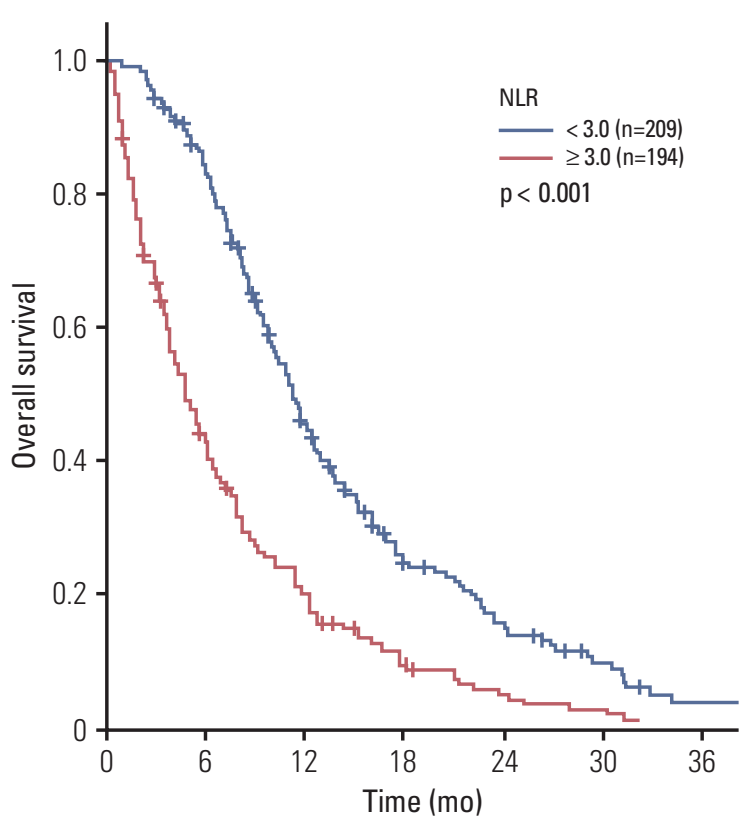

A
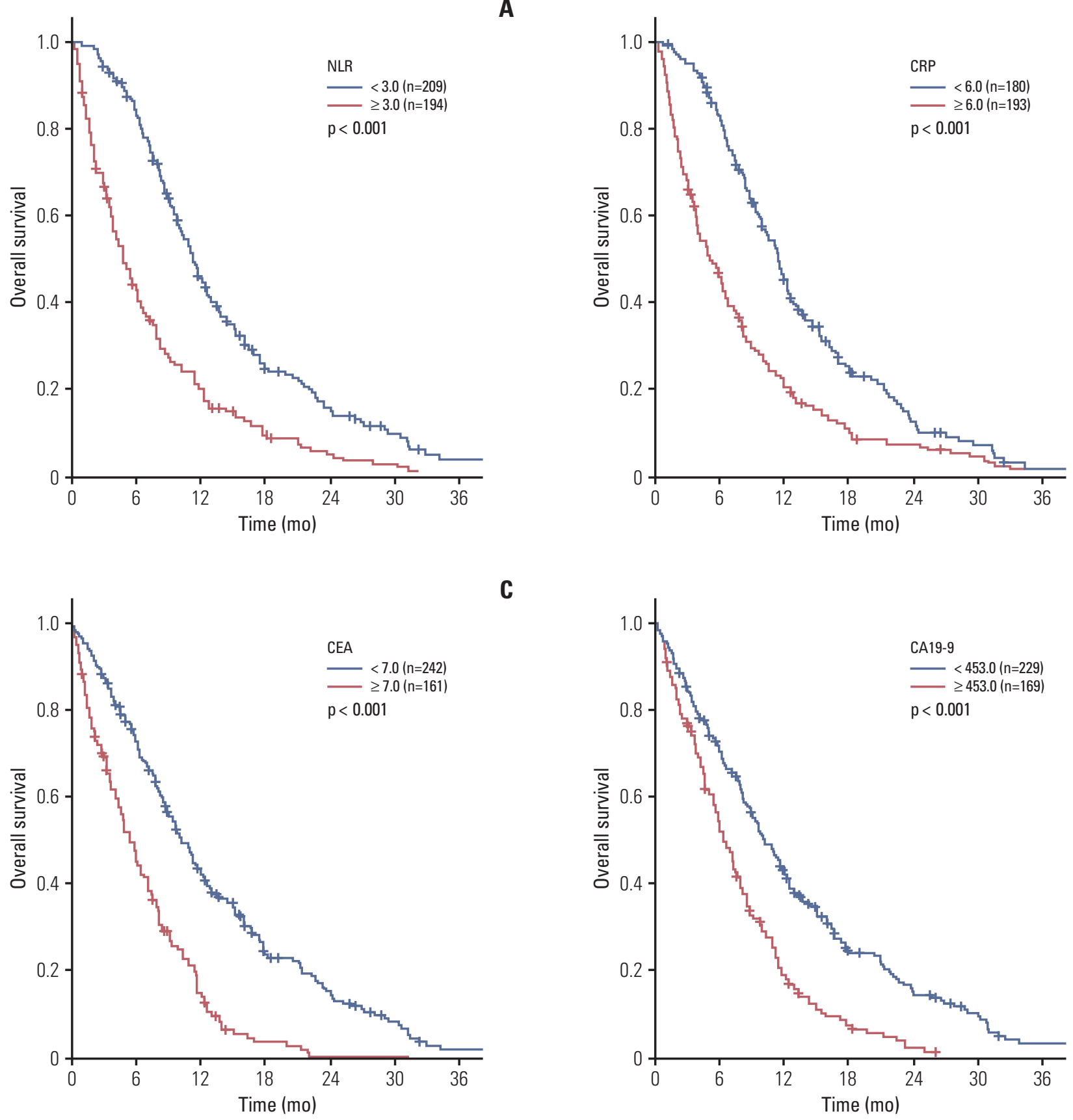

C

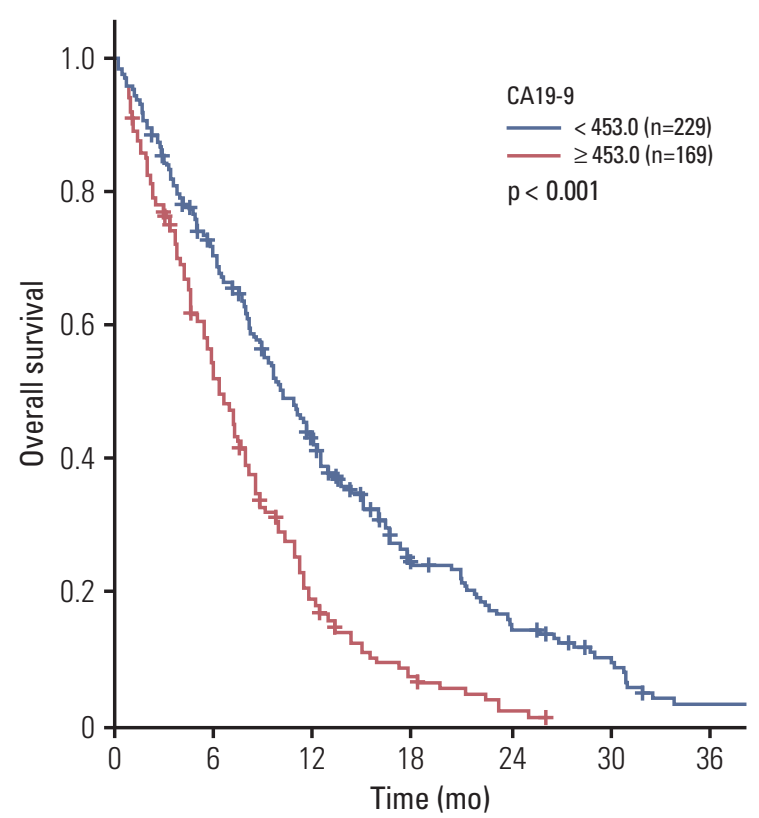

Fig. 1. Kaplan-Meier curves of overall survival for all patients $(n=403)$ according to neutrophil-to-lymphocyte ratio (NLR) (A), C-reactive protein (CRP) (B), carcinoembryonic antigen (CEA) (C), and carbohydrate antigen 19-9 (CA 19-9) (D).

In univariate analysis, age, previous pancreatectomy, tumor location, PS, liver metastasis, peritoneal carcinomatosis, BMI, hemoglobin, WBC count, NLR, serum albumin, BUN, ALP, AST, CRP, CEA, and CA 19-9 showed significant association with OS (Table 2). In multivariate analysis, ECOG
PS, hemoglobin, WBC count, NLR, and CEA were significant in both forward and backward stepwise analyses (Table 3). 
Table 2. Univariate analysis for overall survival

\begin{tabular}{|c|c|c|c|}
\hline Factor & No. & $\mathrm{HR}(95 \% \mathrm{CI})$ & p-value \\
\hline \multicolumn{4}{|l|}{ Age (yr) } \\
\hline$<66$ & 198 & 1 & 0.007 \\
\hline$\geq 66$ & 205 & $1.334(1.081-1.646)$ & \\
\hline \multicolumn{4}{|l|}{ Sex } \\
\hline Male & 198 & 1 & 0.054 \\
\hline Female & 205 & $1.228(0.996-1.514)$ & \\
\hline \multicolumn{4}{|c|}{ Previous pancreatectomy } \\
\hline Yes & 62 & 1 & 0.016 \\
\hline No & 341 & $1.454(1.073-1.972)$ & \\
\hline \multicolumn{4}{|l|}{ Tumor location } \\
\hline Head & 226 & 1 & 0.004 \\
\hline Body/Tail & 177 & $1.369(1.108-1.691)$ & \\
\hline \multicolumn{4}{|c|}{ ECOG performance status } \\
\hline $0-1$ & 217 & 1 & $<0.001$ \\
\hline 2 & 127 & $1.728(1.366-2.185)$ & \\
\hline $3-4$ & 59 & $4.991(3.673-6.782)$ & \\
\hline \multicolumn{4}{|l|}{ Diabetes mellitus } \\
\hline No & 260 & 1 & 0.941 \\
\hline Yes & 143 & $1.008(0.811-1.254)$ & \\
\hline \multicolumn{4}{|l|}{ Liver metastasis } \\
\hline No & 225 & 1 & $<0.001$ \\
\hline Yes & 178 & $1.46(1.182-1.805)$ & \\
\hline \multicolumn{4}{|c|}{ Peritoneal carcinomatosis } \\
\hline No & 324 & 1 & $<0.001$ \\
\hline Yes & 79 & $2.09(1.618-2.7)$ & \\
\hline \multicolumn{4}{|l|}{ Thromboembolism } \\
\hline No & 370 & 1 & 0.099 \\
\hline Yes & 33 & $1.36(0.944-1.959)$ & \\
\hline \multicolumn{4}{|l|}{ BMI $\left(\mathrm{kg} / \mathrm{m}^{2}\right)$} \\
\hline$\geq 18.5$ & 342 & 1 & 0.006 \\
\hline$<18.5$ & 54 & $1.52(1.125-2.054)$ & \\
\hline \multicolumn{4}{|l|}{ Hemoglobin (g/dL) } \\
\hline Normal ( $\geq 12$ ) & 237 & 1 & $<0.001$ \\
\hline Abnormal $(<12)$ & 166 & 1.601 (1.292-1.983) & \\
\hline \multicolumn{4}{|l|}{ WBC count $\left(\times 10^{3} / \mu \mathrm{L}\right)$} \\
\hline Normal $(<10.8)$ & 356 & 1 & $<0.001$ \\
\hline Abnormal ( $\geq 10.8)$ & 47 & $2.936(2.15-4.009)$ & \\
\hline \multicolumn{4}{|l|}{ NLR } \\
\hline$<3$ & 209 & 1 & $<0.001$ \\
\hline$\geq 3$ & 194 & $2.254(1.823-2.788)$ & \\
\hline \multicolumn{4}{|l|}{ Protein $(\mathrm{g} / \mathrm{dL})$} \\
\hline Normal $(\geq 6.9)$ & 172 & 1 & 0.884 \\
\hline Abnormal $(<6.9)$ & 230 & $1.016(0.822-1.256)$ & \\
\hline \multicolumn{4}{|l|}{ Albumin (g/dL) } \\
\hline Normal $(\geq 3.4)$ & 353 & 1 & $<0.001$ \\
\hline Abnormal $(<3.4)$ & 50 & $2.1(1.542-2.86)$ & \\
\hline \multicolumn{4}{|l|}{ Bilirubin (mg/dL) } \\
\hline Normal $(<1.2)$ & 285 & 1 & 0.852 \\
\hline Abnormal ( $\geq 1.2)$ & 118 & $0.978(0.778-1.231)$ & \\
\hline
\end{tabular}


Table 2. Continued

\begin{tabular}{|c|c|c|c|}
\hline Factor & No. & HR $(95 \%$ CI $)$ & p-value \\
\hline \multicolumn{4}{|l|}{ BUN (mg/dL) } \\
\hline Normal $(<23.0)$ & 363 & 1 & 0.006 \\
\hline Abnormal $(\geq 23.0)$ & 39 & $1.638(1.153-2.327)$ & \\
\hline \multicolumn{4}{|l|}{$\operatorname{ALP}(\mathrm{IU} / \mathrm{L})$} \\
\hline Normal (<123) & 226 & 1 & $<0.001$ \\
\hline Abnormal ( $\geq 123)$ & 172 & 1.579 (1.276-1.952) & \\
\hline \multicolumn{4}{|l|}{ AST (IU/L) } \\
\hline Normal $(<30)$ & 219 & 1 & 0.006 \\
\hline Abnormal $(\geq 30)$ & 183 & $1.34(1.086-1.653)$ & \\
\hline \multicolumn{4}{|l|}{ ALT (IU/L) } \\
\hline Normal $(<33)$ & 237 & 1 & 0.893 \\
\hline Abnormal ( $\geq 33$ ) & 165 & $0.986(0.796-1.219)$ & \\
\hline \multicolumn{4}{|l|}{ CRP (mg/L) } \\
\hline$<6$ & 180 & 1 & $<0.001$ \\
\hline$\geq 6$ & 193 & $1.88(1.512-2.336)$ & \\
\hline \multicolumn{4}{|l|}{ CEA (ng/mL) } \\
\hline$<7$ & 242 & 1 & $<0.001$ \\
\hline$\geq 7$ & 161 & $2.332(1.866-2.915)$ & \\
\hline \multicolumn{4}{|l|}{ CA $19-9(\mathrm{U} / \mathrm{mL})$} \\
\hline$<453$ & 229 & 1 & $<0.001$ \\
\hline$\geq 453$ & 169 & $1.927(1.545-2.405)$ & \\
\hline
\end{tabular}

HR, hazard ratio; CI, confidence interval; ECOG, Eastern Cooperative Oncology Group; BMI, body mass index; WBC, white blood cell; NLR, neutrophil-lymphocyte ratio; BUN, blood urea nitrogen; ALP, alkaline phosphatase; AST, aspartate aminotransferase; ALT, alanine aminotransferase; CRP, C-reactive protein; CEA, carcinoembryonic antigen; CA 19-9, carbohydrate antigen 19-9.

Table 3. Multivariate analysis for overall survival

\begin{tabular}{|c|c|c|c|}
\hline Factor & $\chi^{2}$ score & HR $(95 \%$ CI) & p-value \\
\hline \multicolumn{4}{|c|}{ ECOG performance status } \\
\hline $0-1$ & & 1 & $<0.001$ \\
\hline 2 & 11.2 & $1.53(1.19-1.96)$ & \\
\hline $3-4$ & 54.2 & $3.60(2.56-5.06)$ & \\
\hline \multicolumn{4}{|c|}{ Hemoglobin $(\mathrm{g} / \mathrm{dL})$} \\
\hline$\geq 12$ & & 1 & 0.008 \\
\hline$<12$ & 7.0 & $1.36(1.08-1.70)$ & \\
\hline \multicolumn{4}{|c|}{ WBC count $\left(\times 10^{3} / \mu \mathrm{L}\right)$} \\
\hline$<10.8$ & & 1 & 0.002 \\
\hline$\geq 10.8$ & 9.6 & $1.77(1.23-2.55)$ & \\
\hline \multicolumn{4}{|l|}{ NLR } \\
\hline$<3$ & & 1 & $<0.001$ \\
\hline$\geq 3$ & 21.0 & $1.78(1.39-2.28)$ & \\
\hline \multicolumn{4}{|c|}{ CEA (ng/mL) } \\
\hline$<7$ & & 1 & $<0.001$ \\
\hline$\geq 7$ & 50.9 & $2.43(1.90-3.10)$ & \\
\hline
\end{tabular}

HR, hazard ratio; CI, confidence interval; ECOG, Eastern Cooperative Oncology Group; WBC, white blood cell; NLR, neutrophil-lymphocyte ratio; CEA, carcinoembryonic antigen. 


\section{Prognosis scoring system}

A prognostic system was devised using these five independent prognostic factors. Scores were calculated by summing the rounded chi-squre scores of these factors (Table 4): $2($ ECOG PS $=2)$ or 11 (ECOG PS $\geq 3)+1$ (hemoglobin $<12 \mathrm{~g} /$ $\mathrm{dL})+2($ WBC count $\geq 10,800 / \mu \mathrm{L})+4(\mathrm{NLR} \geq 3.0)+10(\mathrm{CEA} \geq$ $7 \mathrm{ng} / \mathrm{mL}$ ). The scores ranged from 0 to 28 , and the maximally discriminative cut-off values were 6 and 25, respectively.

Patients were then categorized according to three subgroups based on their scores: low (198 [49.1\%]), intermediate (182 [45.2\%]), and high-risk groups (23 [5.7\%]). The median OS was 11.7 months (95\% CI, 10.2 to 13.2), 6.2 months (95\% CI, 5.0 to 7.4 ), and 1.3 months (95\% CI, 0.9 to 1.7$)$, respectively $(\mathrm{p}<0.001)$ (Fig. 2). One-year survival rate was 49.0\%, 18.9\%, and $0 \%$, respectively. The internally validated Harrell's c-index of the scoring system was 0.731 (95\% CI, 0.696 to $0.766)$.

\section{Association with treatment outcome}

Of the 403 patients, $315(78.2 \%)$ underwent palliative chemotherapy and $88(21.8 \%)$ refused chemotherapy, receiving supportive care only. Palliative chemotherapy included gemcitabine monotherapy $(21.6 \%)$, gemcitabine doublet $(40.4 \%)$, and fluoropyrimidine-based chemotherapy $(8.9 \%)$. Radiotherapy was performed with palliative aim in $7.2 \%$ of patients. With these different kinds of chemotherapy regimens, there were no significant differences in OS.

Patients receiving chemotherapy had significantly longer survival than patients who received best supportive care only (median OS, 10.3 vs. 2.5 months; $\mathrm{p}<0.001$ ). One hundred seventy-five $(88.4 \%), 138(75.8 \%)$, and only two patients $(8.7 \%)$ in the low, intermediate, and high-risk groups received chemotherapy, respectively. Palliative chemotherapy provided patients with a survival benefit in the low-risk group (median OS, 12.5 months vs. 5.9 months; $p<0.001$ ) (Fig. 3A), and patients in the intermediate-risk group also benefitted from chemotherapy (median OS, 8.0 vs. 2.0 months; $\mathrm{p}<0.001$ ) (Fig. 3B). However, no survival difference was observed with chemotherapy in the high-risk group (median OS, 3.7 vs. 1.2 months; p=0.163) (Fig. 3C).

\section{Discussion}

Pancreatic cancer is the only human malignancy for which patients' survival has rarely improved during the past 30 years. Chemotherapy remains the mainstay of treatment in metastatic pancreatic cancer. However, current systemic
Table 4. Components of the prognostic prediction score for pancreatic ductal adenocarcinoma

\begin{tabular}{lr} 
Factor & Score \\
ECOG performance status & \\
$0-1$ & 0 \\
2 & +2 \\
$3-4$ & +11 \\
Hemoglobin $(\mathrm{g} / \mathrm{dL})$ & \\
$\geq 12$ & 0 \\
$<12$ & +1 \\
WBC count $\left(\times 10^{3} / \mu \mathrm{L}\right)$ & 0 \\
$<10.8$ & +2 \\
$\geq 10.8$ & \\
NLR & 0 \\
$<3$ & +4 \\
$\geq 3$ & \\
CEA (ng/mL) & 0 \\
$<7$ & +10 \\
\hline 7 &
\end{tabular}

ECOG, Eastern Cooperative Oncology Group; WBC, white blood cell; NLR, neutrophil-lymphocyte ratio; CEA, carcinoembryonic antigen.

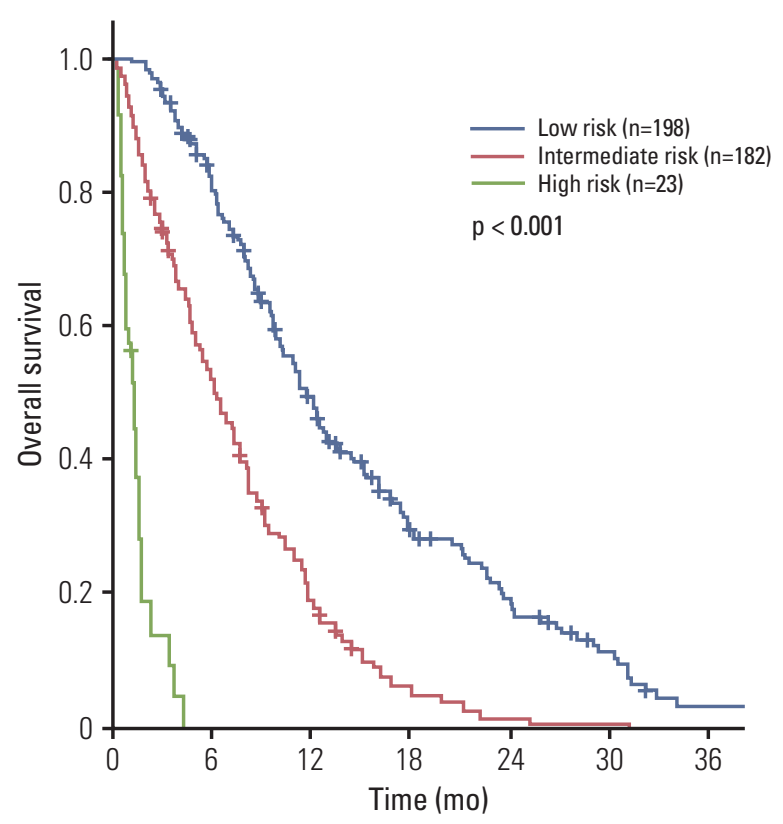

Fig. 2. Kaplan-Meier overall survival curves according to risk group. Patients were classified according to three risk groups based on prognostic prediction score: low risk, 0-6; intermediate risk, 7-25; and high risk, 26-28. 

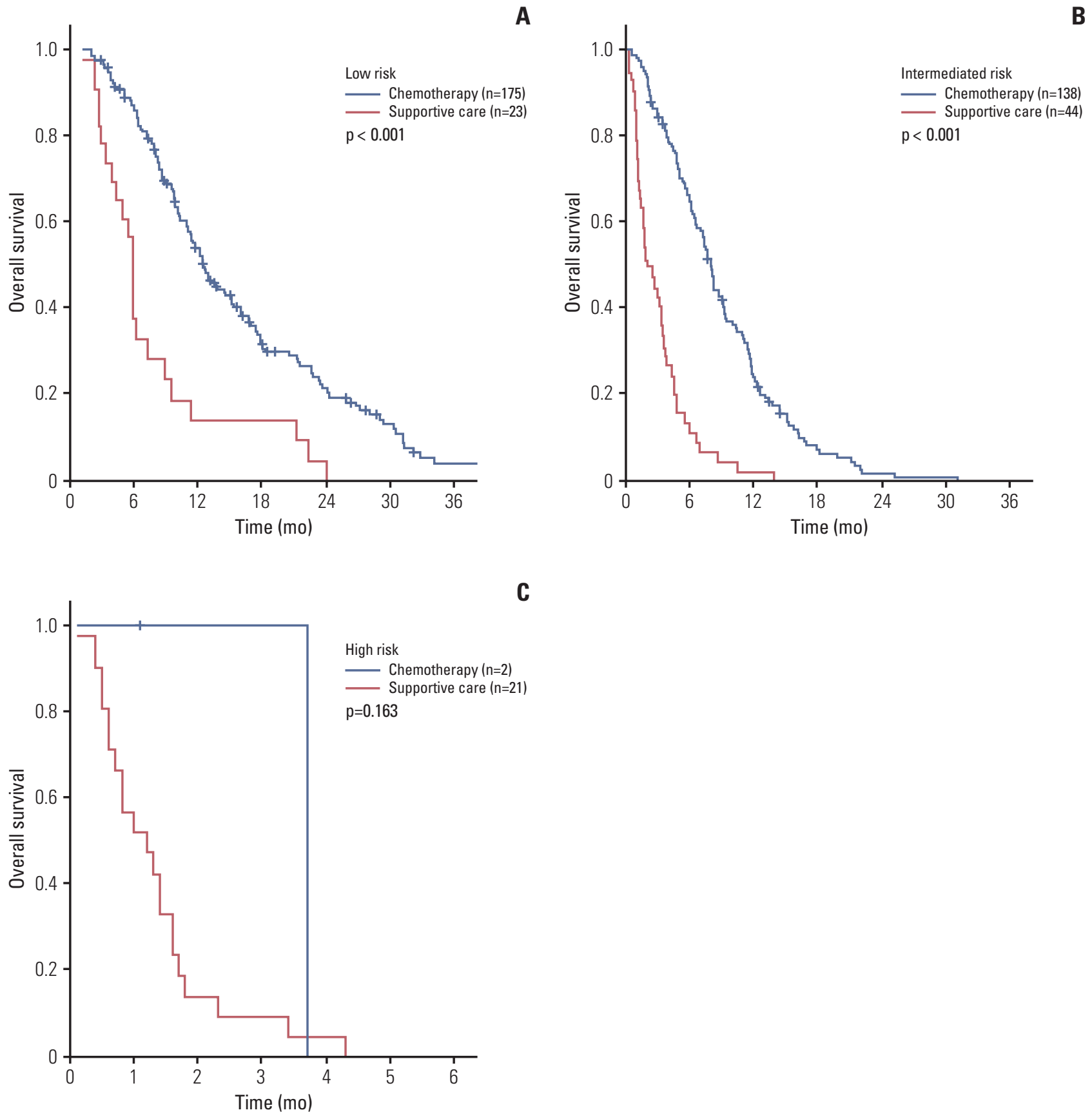

Fig. 3. Kaplan-Meier overall survival survival curves according to palliative treatment in the low-risk (A), intermediate-risk (B), and high-risk (C) groups.

treatments offer only a modest benefit in tumor-related symptoms and survival and these benefits, if any, are usually limited to patients—even with a novel regimen of FOLFIRINOX—with good PS and adequate nutritional status, and abrupt changes in clinical status may occur in debilitated patients with advanced disease despite systemic treatment. Therefore, primary point was (1) how to predict survival of patients at the time of initial diagnosis and (2) how to differentiate patients who might benefit from chemotherapy from those cannot. 
In general, pancreatic cancer patients are not clinically separated into prognostic groups, with the exception of surgical status, before treatment. Therefore, limited studies have focused on metastatic disease [3,4]. A previous Korean study reported liver metastasis, peritoneal seeding, high CRP, and low serum albumin as poor indicators of OS. A western study reported age, weight loss, and metastasis as poor prognostic factors. Both studies focused on gemcitabine-treated patients, and are small retrospective analyses with questionable statistical powers. However, pancreatic cancer is a heterogeneous disease and recent introduction of the three-drug combination regimen or the new taxane represent key advances for better control of the disease over gemcitabine, which requires a new prognostic model.

Another debate was cutoff points of routinely measured tumor markers-CRP, CEA, and CA 19-9 [9]. Using a chemiluminescence immunoassay, the standard diagnostic cutoff points for CA 19-9 and CEA were $37 \mathrm{IU} / \mathrm{mL}$ and $5 \mathrm{ng} / \mathrm{mL}$, respectively [10]. However, the cut-off points for these variables have not been established in metastatic disease. Some researchers advocate that CA 19-9 serum level of $<100$ $\mathrm{U} / \mathrm{mL}$ implies likely resectable disease, while levels $>100$ $\mathrm{U} / \mathrm{mL}$ may suggest unresectablity or metastatic disease [11]. However, most studies used median values or normal ranges for dichotomization. For dichotomizing continuous variables, splits of some percentile or average are arbitrary and may not be useful in assessing the true prognostic value of variables [12]. In our study, instead, cutoff point was derived using the Contal and O'Quigley method, which selects the cut-off point that maximizes the model likelihood only after all possible cut-offs have been evaluated [6]. In addition, K-adaptive partitioning for the survival model was used to determine minimum p-value.

The purpose of implementing a scoring system is for clinical decision-making and patient risk stratification, using routinely measurable clinical parameters on admission. In this study PS, hemoglobin, WBC, NLR, and CEA were identified as independent prognostic markers. For patients with a score of $\leq 6$, we may hypothesize that their survival can be extended up to 12.5 months by chemotherapy, which provides us with a rationale for recommendation of relatively aggressive treatment such as FOLFIRINOX. Conversely, high-risk group (score $>25$ ) patients had an extremely poor prognosis, with a median survival duration of only 1.3 months and the 1-year survival rate was less than $1 \%$. Chemotherapy has no statistical benefit in these patients, but it is impetuous for now to declare that chemotherapy is contraindicated for the high risk group, in which only two patients who received chemotherapy were included for analysis. Intermediate-risk patients showed comparable outcomes with gemcitabine-based treatment. Thus, we can recommend conventional gemcitabine-based therapy to these patients. Ultimately, these findings suggest that this scoring system can be helpful in selection of the "right" patients for the "right" treatment.

Several previous studies reported reduced survival in patients with elevated CA 19-9 levels [13]. However, CA 19-9 was not included in our system. We believe increased false positive results in the presence of obstructive jaundice and inflammation-20\% of our patients accompanied obstructive jaundice and $12 \%$ with systemic leucocytosiscould severely limit the universal applicability of serum CA 19-9 levels in pancreatic cancer management. Instead, our study showed that NLR, leukocytosis, and CEA were independent. Hypothesis of the role of inflammation in development and prognosis of cancer has been examined and several studies have suggested that NLR and WBC count are indicative of survival $[3,14]$. Whether the systemic inflammation is malignancy-associated or is the result of any co-morbid conditions that cancer patients may suffer is still unclear, but here we assumed that systemic inflammation might also occur in commonly prevalent conditions. One plausible explanation is that cellular response of blood components might be mediated through endothelial dysfunction, leading to depletion of vasodilator, antithrombotic, and anti-atherogenic properties of the endothelium and may result in capillary leukocytosis and subsequent increased vascular resistance [15]. In addition, high neutrophil counts reflect inflammation, whereas low lymphocyte counts reflect malnutrition [16]. Cytokine-mediated systemic inflammation has been shown to promote angiogenesis, DNA damage response, and tumor invasion [17]. NLR has recently been reported as a prognostic marker in colorectal and lung cancers $[14,15]$, and its prognostic value in pancreatic cancer has also been reported [18]. Although an NLR cut-off value of 4.0 or 5.0 has been shown to have significant prognostic value in resectable cancers, a cutoff point of 3.0 may be reasonable for survival predication, which is consistent with a previous report [18-20].

The significance of CEA in pancreatic cancer has been rarely investigated [3]. CEA functions as an adhesion molecule during metastasis, and Hostetter et al. [21] reported that CEA increases the percentage of colorectal liver metastases from $2 \%$ to $48 \%$ in vivo. In a small study, CEA was identified as prognostic in advanced pancreatic cancer; superior survival outcome has been reported in patients with low CEA levels $(<2.5 \mathrm{ng} / \mathrm{mL})$ compared to those with high CEA levels [22]. Although the cut-off value for CEA in our study was 7 $\mathrm{ng} / \mathrm{mL}$, further evaluation is warranted to elucidate the role of CEA in pancreatic cancer. Another factor, hemoglobin level, has been reported as a poor prognostic factor and is reflective of nutrition or general condition [23].

However, a prognostic model using clinical parameters only had a limitation in that tumor biology could not be 
reflected. In pancreatic cancer, K-ras mutations occur in $90 \%$ of cases, and overexpression of growth factors such as epidermal growth factor, transforming growth factor $\alpha$ is common [24]. In addition, expression of these growth factors and their receptor has been found to show association with decreased survival, thus, a combined approach using both clinical parameters and biologic markers is warranted for implementation of a more robust prognostic model. However, our system showed a Harrell's c-index of 0.731, indicating sufficient discrimination power for patients according to the risk, and it warrants prospective clinical utility [25]. Another limitation of this study is the retrospective and nonrandomized design. Patients underwent heterogeneous treatments including gemicitabine monotherapy or doublet, fluoropyrimidines, oral chemotherapy or even concurrent chemoradiotherapy which might have affected differential influence on survival.

\section{Conclusion}

In conclusion, we found that NLR and CEA were independent prognostic factors and suggest 3.0 for NLR and $7 \mathrm{ng} / \mathrm{mL}$ for CEA as statistically determinant cut-off values for prognostic dichotomization. In addition, our prognostic scoring system provides rationale for risk stratification of advanced PDAC patients, leading to individualized treatment. Further studies are needed to evaluate the accuracy of this scoring system.

\section{Conflicts of Interest}

Conflict of interest relevant to this article was not reported.

\section{Acknowledgments}

This study was supported by a grant from the National R\&D Program for Cancer Control, Ministry of Health \& Welfare, Republic of Korea (to H.C. Jeung, No. 1420060).

\section{References}

1. Ryan DP, Hong TS, Bardeesy N. Pancreatic adenocarcinoma. N Engl J Med. 2014;371:2140-1.

2. Ni XG, Bai XF, Mao YL, Shao YF, Wu JX, Shan Y, et al. The clinical value of serum CEA, CA19-9, and CA242 in the diagnosis and prognosis of pancreatic cancer. Eur J Surg Oncol. 2005;31:164-9.

3. Stocken DD, Hassan AB, Altman DG, Billingham LJ, Bramhall SR, Johnson PJ, et al. Modelling prognostic factors in advanced pancreatic cancer. Br J Cancer. 2008;99:883-93.

4. Yi JH, Lee J, Park SH, Lee KT, Lee JK, Lee KH, et al. A prognostic model to predict clinical outcomes with first-line gemcitabine-based chemotherapy in advanced pancreatic cancer. Oncology. 2011;80:175-80.

5. Ciela B. Hematology in practice. 2nd ed. Philadelphia, PA: F.A. Davis Company; 2011.

6. Contal C, O'Quigley J. An application of changepoint methods in studying the effect of age on survival in breast cancer. Comput Stat Data Anal. 1999;30:253-70.

7. Eo SH, Hong SM, Cho HY. K-adaptive partitioning for sur- vival data: the Kaps add-on package for R. arXiv:1306.4615. Ithaca, NY: arXiv, Cornell University Library; 2013.

8. Harrell FE Jr, Lee KL, Matchar DB, Reichert TA. Regression models for prognostic prediction: advantages, problems, and suggested solutions. Cancer Treat Rep. 1985;69:1071-7.

9. Ziske C, Schlie C, Gorschluter M, Glasmacher A, Mey U, Strehl J, et al. Prognostic value of CA 19-9 levels in patients with inoperable adenocarcinoma of the pancreas treated with gemcitabine. Br J Cancer. 2003;89:1413-7.

10. Lee KJ, Yi SW, Chung MJ, Park SW, Song SY, Chung JB, et al. Serum CA 19-9 and CEA levels as a prognostic factor in pancreatic adenocarcinoma. Yonsei Med J. 2013;54:643-9.

11. Ballehaninna UK, Chamberlain RS. Serum CA 19-9 as a biomarker for pancreatic cancer: a comprehensive review. Indian J Surg Oncol. 2011;2:88-100.

12. Mazumdar M, Glassman JR. Categorizing a prognostic variable: review of methods, code for easy implementation and applications to decision-making about cancer treatments. Stat Med. 2000;19:113-32. 
13. Ballehaninna UK, Chamberlain RS. The clinical utility of serum CA 19-9 in the diagnosis, prognosis and management of pancreatic adenocarcinoma: an evidence based appraisal. J Gastrointest Oncol. 2012;3:105-19.

14. Sarraf KM, Belcher E, Raevsky E, Nicholson AG, Goldstraw P, Lim E. Neutrophil/lymphocyte ratio and its association with survival after complete resection in non-small cell lung cancer. J Thorac Cardiovasc Surg. 2009;137:425-8.

15. Walsh SR, Cook EJ, Goulder F, Justin TA, Keeling NJ. Neutrophil-lymphocyte ratio as a prognostic factor in colorectal cancer. J Surg Oncol. 2005;91:181-4.

16. Fock RA, Blatt SL, Beutler B, Pereira J, Tsujita M, de Barros FE, et al. Study of lymphocyte subpopulations in bone marrow in a model of protein-energy malnutrition. Nutrition. 2010;26: 1021-8.

17. Palucka K, Coussens LM, O'Shaughnessy J. Dendritic cells, inflammation, and breast cancer. Cancer J. 2013;19:511-6.

18. Luo G, Guo M, Liu Z, Xiao Z, Jin K, Long J, et al. Blood neutrophil-lymphocyte ratio predicts survival in patients with advanced pancreatic cancer treated with chemotherapy. Ann Surg Oncol. 2015;22:670-6.

19. Garcea G, Ladwa N, Neal CP, Metcalfe MS, Dennison AR, Berry DP. Preoperative neutrophil-to-lymphocyte ratio (NLR) is associated with reduced disease-free survival following curative resection of pancreatic adenocarcinoma. World J Surg.
2011;35:868-72.

20. Sugiura T, Uesaka K, Kanemoto H, Mizuno T, Okamura Y. Elevated preoperative neutrophil-to-lymphocyte ratio as a predictor of survival after gastroenterostomy in patients with advanced pancreatic adenocarcinoma. Ann Surg Oncol. 2013; 20:4330-7.

21. Hostetter RB, Augustus LB, Mankarious R, Chi KF, Fan D, Toth $\mathrm{C}$, et al. Carcinoembryonic antigen as a selective enhancer of colorectal cancer metastasis. J Natl Cancer Inst. 1990;82:380-5.

22. Yasue M, Sakamoto J, Teramukai S, Morimoto T, Yasui K, Kuno N, et al. Prognostic values of preoperative and postoperative CEA and CA19.9 levels in pancreatic cancer. Pancreas. 1994;9:735-40.

23. Ruiz-Tovar J, Martin-Perez E, Fernandez-Contreras ME, Reguero-Callejas ME, Gamallo-Amat C. Identification of prognostic factors in pancreatic cancer. Cir Cir. 2011;79:313-22.

24. Ghaneh P, Kawesha A, Evans JD, Neoptolemos JP. Molecular prognostic markers in pancreatic cancer. J Hepatobiliary Pancreat Surg. 2002;9:1-11.

25. Keam B, Kim DW, Park JH, Lee JO, Kim TM, Lee SH, et al. Nomogram predicting clinical outcomes in non-small cell lung cancer patients treated with epidermal growth factor receptor tyrosine kinase inhibitors. Cancer Res Treat. 2014;46:323-30. 